\title{
Reliability prediction, scatter-band analysis and fatigue limit assessment of high-cycle fatigue properties in EN-GJS700-2 ductile cast iron
}

\author{
Mohammad Jamalkhani Khameneh ${ }^{1}$ and Mohammad Azadi ${ }^{2, *}$ \\ ${ }^{1}$ Faculty of Mechanical Engineering, Semnan University, Semnan, Iran \\ ${ }^{2}$ Faculty of Mechanical Engineering, Semnan University, Semnan, Iran
}

\begin{abstract}
In this article, high-cycle fatigue properties of the EN-GJS700-2 ductile cast iron were experimentally examined, using the four-point rotary-bending fatigue machine. Such material has been widely utilized for manufacturing crankshafts in automotive industries. Therefore, standard specimens were extracted from the crankshaft to consider the manufacturing effect. The other aim of this work is to develop a model, anticipating the probabilistic behavior of the EN-GJS700-2 ductile cast iron. For such objective, the parametric analysis of the most commonly used statistical distributions were performed and compared. Based on the best fitted distribution function on experimental data, fatigue lifetime reliability specifications of the material were analyzed and represented through probabilistic modeling. In addition, the S-N diagram with its scatter-band were conducted at different values of the confidence level. As another investigation, the criterion for designing the crankshaft is the infinite lifetime, or in more practical terms, is the design with allowable stress levels, below the fatigue limit. Based on the staircase test methodology, fatigue endurance limit tests were conducted. Then, statistical trends of the fatigue limit were analyzed by the Dixon-Mood method, based on the maximum likelihood estimation. Obtained results represents that the fatigue limit was $211 \mathrm{MPa}$ for the ductile cast iron.
\end{abstract}

\section{Introduction}

Ductile cast irons have been extensively utilized in crankshafts, as a main part of automotive engines [1]. Such engine components are subjected to the fatigue phenomenon, due to constant amplitude loading, during their usage. On the other hand, fatigue test results (S-N data) usually expose remarkable scatters, even when tests are intently performed to diminish the experimental error [2-4]. As a result of this inherent variation in fatigue data, median S-N fatigue lifetime curves are not adequate for the fatigue analysis in order to design components [4]. Therefore, the statistical analysis through testing activities must be considered to evaluate the fatigue property of such material, for the design of the engine crankshaft, which are subjected to the high-cycle fatigue (HCF) damage.

In this field of study, several researches have been implemented by researchers. Caiza and Ummenhofer [5] introduced a procedure to model S-N curves, subject to the Weibull model. They presented two applications, considering experimental data of girders and welded specimens, made of the S690QL steel. They obtained that estimated S-N curves, through the Weibull model onto HCF or very HCF regions, were more precise than those obtained by applying the Basquin model. Chandrappa and Biligiri [6] investigated the effect of stress levels and frequencies on the fatigue behavior of pervious concrete paving mixtures. Non-parametric and parametric methods showed that the fatigue lifetime were significantly affected by the stress level and the stiffness, compared to the loading frequency in the range of 2-10 Hz. KaplanMeier survival curves demonstrated a sudden drop in the survival proportion at higher stress levels. They also fitted three-parameter Weibull and log-normal distributions to fatigue data, where the log-normal distribution performed a better fitness. Zhu et al. [7] represented a probabilistic approach for modeling the damage accumulation to investigate the fatigue reliability of railway axle steels. They performed the fatigue reliability analysis under variable amplitude loadings. They acquired an agreement between predicted results and experimental measurements under different loading conditions. Feng et al. [8] evaluated mechanical properties, fatigue properties and damage characteristics of the rubber-modified recycled aggregate concrete. They conducted the statistical analysis on test results, using the Weibull distribution and also suggested double logarithm fatigue equations for the material at different failure probabilities. Chen et al. [9] investigated the crankshaft fatigue phenomenon, using the experimental method. In addition, they performed the statistical regression analysis of eight hypothesis distributions, based on experiment data and evaluated the degree of the fitting effect, between experimental results with chosen statistical models.

* Corresponding author: $\underline{m \text { azadi } @ \text { semnan.ac.ir }}$ 
Finally, they discovered that the three-parameter Weibull distribution model fitted data, in a superior condition.

Borgia et al. [10] investigated a test methodology based on accelerated lifetime tests, capable to evaluate the reliability of a domestic washing machine. They utilized two-parameter Weibull, as a probability function, to assess the reliability of the machine, at each overstress level. Cevik and Gurbuz [11] evaluated the effect of fillet rolling on the fatigue behavior of the EN-GJS800-2 ductile cast iron crankshaft, with the application of diesel engines. They used the staircase test methodology to demonstrate $\mathrm{S}-\mathrm{N}$ curves and to measure fatigue endurance limits. They also revealed that induced compressive residual stresses increased the surface hardness and increased the dislocation density, which provided an improvement in the fatigue strength. Zhai and $\mathrm{Li}$ [3] performed a conditional probability density distribution surface, by two functions such as a function of the mean value of the population and the stress level and the other one was a function of the standard deviation and the stress level. They also proposed a theoretical fatigue limit, according to the small probability event.

Klemenc and Fajdiga [12] presented an estimation of the $\mathrm{S}-\mathrm{N}$ curve and its scatter, by applying the two-parameter Weibull distribution to demonstrate the scatter of the lifetime, at a stress level. They described the S-N curve and its scatter, by three parameters, including the constant term, the scale coefficient and the shape parameter of the Weibull distribution. Mohd et al. [13] evaluated scatter characteristics of the fatigue lifetime, by a threeparameter Weibull distribution in a die-cast magnesium alloy. They also investigated the correlation between the scatter behavior of the fatigue lifetime and the pore size, observed on the cross section, as well as on the fracture surface. They concluded that the three-parameter Weibull distribution was suitable to describe the scatter behavior of both the fatigue lifetime and the pore size. Rathod et al. [14] presented a methodology for modeling the probabilistic distribution of the fatigue damage accumulation for single- and multi-stress level loading. The methodology used the linear damage accumulation model of Palmgren-Miner, as a probabilistic S-N curve, and an approach for a one-to-one transformation of probability density functions. Then, they predicted the reliability by the proposed methodology, using the dynamic statistical model of the cumulative fatigue damage.

Bouraoui et al. [15] developed a probabilistic approach to investigate the HCF behavior reliability of shot peened metallic components. They evaluated the reliability in different values, using the strength-load method and computed by the numerical Monte-Carlo simulation. Then, shot peening HCF reliability predictions and obtained iso-probabilistic Crossland diagrams showed a good agreement with experimental results. Sghaier et al. [16] presented a methodology to predict the reliability of metallic parts, according to their HCF behavior. They utilized the multi-axial Crossland criterion, in the case of normally distributed in-phase fully reversed torsion and bending loadings and HCF material characteristic parameters. Their results showed that the proposed method was qualitatively consistent with physical observations. Spiteri et al. [17] evaluated the fatigue limit of rolled ductile cast iron crankshafts, under bending. They used the staircase test methodology to find the fatigue strength of the material.

Schijve [18] compared three statistical distribution functions, containing the normal distribution, the threeparameter Weibull distribution and the three-parameter normal distribution for the logarithmic lifetime. The second and the third function showed a good agreement of fitted data of 30 similar test results. Khashaba [19] performed rotating bending fatigue tests on glass fiber reinforced polyester composites. He conducted the statistical analysis of experimental fatigue lifetime results, using the two-parameter Weibull distribution and calculated the safe design of the lifetime, based on the time to the first failure concept for $99 \%$ of the confidence level and at two values of the reliability, 0.368 and 0.990 . $\mathrm{He}$ concluded that these values of the reliability and the confidence level were appropriate and could be utilized to predict the flexural fatigue lifetime of such composites. Shen [20] developed a probability-based methodology to predict the reliability of gas turbine engine blades, exposed to HCF loadings. Doremus [21] compared three statistical distribution functions such as normal, Weibull and extreme value distributions, to fit glass fracture strengths. He concluded that the accuracy of the extreme value distribution was much better than those of others.

A review of literatures has indicated that the assessment of statistical fatigue problems of structures and materials has been mentioned in numerous publications. In addition, it is concluded that in most cases, Weibull and normal distributions have been used for describing the reliability. However, investigating the reliability and the fatigue limit of crankshafts is still rare. To bridge this gap, in this article, the reliability of the EN-GJS700-2 ductile cast iron has been predicted. For such objective, several statistical distributions have been investigated and compared. The article continues with evaluating the endurance limit, according to the staircase test methodology. Then, obtained results have been presented in figures, including probability, reliability and the S-N curve with its scatter-band.

\section{Materials, Fatigue Testing and Statistical Analysis}

\subsection{Studied materials and experiments}

In this research, the studied material was a ductile cast iron, entitled EN-GJS700-2, which has been utilized widely for manufacturing engine crankshafts in automotive industries [1]. The chemical composition of such material (obtained by crankshaft webs) is shown in Table 1. The microstructure of the studied material has been comprehensively investigated in the previous work [1].

Tests were carried out, under fully reversed bending loading, by a four-point rotary-bending high-cycle fatigue (HCF) testing machine. Related equipments including the picture of the fatigue testing machine and its components, besides the real specimen and the geometry of standard 
specimens were demonstrated in the literature [1]. Sinusoidal waves with a frequency of $100 \mathrm{~Hz}$ and a stress ratio $\left(R_{\sigma}\right)$ of -1 (the fully reversed condition), was utilized for fatigue tests, at the room temperature of $25^{\circ} \mathrm{C}$. It should be noted that the loading frequency has been chosen base on the rated power condition (equal to highest mechanical loads), about $6000 \mathrm{rpm}$ of the engine speed. The applied force for stress amplitude levels was considered as $110,100,90,80,70,60,55$ and $50 \mathrm{~N}$. It should be added that the roughness of machined specimens was measured and compared with standards in the literature [1]. As a recommendation in references [4, 22], the sample number for the fatigue test to generate the S-N curve, should be in the range of 12-24 specimens. Given this, the S-N curve was characterized by testing 24 specimens, 4 specimens at each stress level (6 different levels of stress amplitude) and the fatigue endurance limit was denoted by the staircase test methodology, with 7 specimens. The total number of specimens was 31 samples, which were extracted from engine crankshafts.

Table 1. The chemical composition (wt.\%) of the ductile cast iron, entitled EN-GJS700-2.

\begin{tabular}{ccccccc}
\hline $\mathrm{C}$ & $\mathrm{Si}$ & $\mathrm{Mn}$ & $\mathrm{Cu}$ & $\mathrm{Ni}$ & $\mathrm{P}$ & $\mathrm{Sn}$ \\
\hline 3.50 & 2.18 & 0.45 & 0.48 & 0.02 & 0.014 & 0.050 \\
\hline $\mathrm{Cr}$ & $\mathrm{Al}$ & $\mathrm{Ti}$ & $\mathrm{V}$ & $\mathrm{W}$ & $\mathrm{Co}$ & $\mathrm{Fe}$ \\
\hline 0.030 & 0.016 & 0.035 & 0.003 & 0.002 & 0.004 & based \\
\hline
\end{tabular}

\subsection{Statistical distribution functions and fatigue reliability}

Good understanding of the statistical behavior for fatigue properties of the ductile cast iron is necessary for the design of engine crankshafts, due to the importance of the component performance. On the other hand, to predict the reliability (the probability of survivals) of such material, it is essential to discover the suitable probability function that fitted well on data, collected at each stress level. Therefore, the statistical analysis of experimental fatigue lifetime results was investigated, utilizing six common distribution functions such as Weibull (two- and threeparameter), normal, log-normal, extreme maximum value and smallest extreme value distributions. These distribution functions were characterized by a probability density function (PDF), $f(t)$, and the associated cumulative distribution function (CDF), $F(t)$ for failures and $R(t)$ for non-failures. In this case study, $t$ (random variable) refers to the fatigue lifetime. Probability functions of such distributions are shown in Table 2.

The PDF, $f(t)$, specifies the distribution of a failure through the fatigue lifetime and indicates the absolute failure speed [23]. In addition, the probability that a test sample will fail during an interval, $[0, t]$, is defined by the CDF, $F(t)$, which could be found as follows, [23],

$$
F(t)=\int_{0}^{t} f(t) d t
$$

In addition, the probability that a test sample will survive during an interval $[0, t]$ (the probability of non-failure) could be derived by the CDF, $R(t)$, called as "reliability" and is given by the following relation, [23],

$$
R(t)=1-F(t)
$$

The probability plot and the maximum likelihood estimator (MLE) was employed in the MINITAB statistical software program to perform the distribution fitting process. The MLE method is a statistical procedure for the parameter estimation by maximizing the likelihood function. This method is recommended, since maximum likelihood estimations often have desirable statistical properties [4].

Table 2. Probability formulations for six distribution functions.

\begin{tabular}{ccc}
\hline Distributions & $f(t)$ & $F(t)$ \\
\hline $\begin{array}{c}\text { Two-parameter } \\
\text { Weibull } \\
\text { Three- } \\
\text { parameter } \\
\text { Weibull }\end{array}$ & $\frac{\beta}{\theta}\left(\frac{t}{\theta}\right)^{\beta-1} e^{-\left(\frac{t}{\theta}\right)^{\beta}}$ & $1-e^{-\left(\frac{t}{\theta}\right)^{\beta}}$ \\
Normal & $\left.\frac{1}{\theta}\right)^{\beta-1} e^{-\left(\frac{t-\gamma}{\theta}\right)^{\beta}}$ & $1-e^{-\left(\frac{t-\gamma}{\theta}\right)^{\beta}}$ \\
Log-normal & $\frac{1}{\sqrt{2 \pi} \sigma t} e^{\left(-\frac{(t-\mu)^{2}}{2 \sigma^{2}}\right)}$ & $\emptyset\left(\frac{t-\mu}{\sigma}\right)$ \\
$\begin{array}{c}\text { Extreme } \\
\text { maximum value } \\
\text { Smallest } \\
\text { extreme value }\end{array}$ & $\frac{1}{\sigma} e^{\left(-\frac{t-\mu}{\sigma}\right)} e^{\left(-\exp \left(-\frac{t-\mu}{\sigma}\right)\right)}$ & $\varnothing\left(\frac{\ln (t)-\mu}{\sigma}\right)$ \\
\hline
\end{tabular}

For two- and three-parameter Weibull functions, mentioned in Table 2, $\beta$ is the shape parameter. Then, $\theta$ is the characteristic lifetime, which is also called the scale parameter. The three-parameter Weibull function has an additional parameter, $\gamma$, which is called the location parameter [23]. In addition, in four other functions, $\mu$ is the location parameter (the mean value of experimental data at each stress level) and $\sigma$ is the scale parameter (the variance of experimental data at each stress level). The function $\varnothing(t, \mu, \sigma)$ indicates the standard normal CDF [23]. The value of above parameters in six distribution functions is denoted by rewriting PDFs in the form of the standard linear equations through a mathematical transformation: $Y=A+B T \quad[9,19]$. These linear equations of six distribution functions with their variables, $Y$ and $T$, are illustrated in Table 3. Values of $A$ and $B$ in all linear equations of distributions have been specified by following equations, using the MLE [22]:

$$
\hat{B}=\frac{\hat{A}=\bar{Y}-B \bar{T}}{\sum_{i=1}^{k}\left(T_{i}-\bar{T}\right)\left(Y_{i}-\bar{Y}\right)}
$$

The symbol "over-bar" denotes the average value and $k$ is the total number of specimens in each stress level [22].

Table 3. Standard linear equations of six PDFs and variables of

\begin{tabular}{ccc}
\multicolumn{3}{c}{$Y=A+B T}$. \\
\hline Distributions & $Y$ & $T$ \\
\hline Two-parameter & $\ln \left(\ln \left(\frac{1}{1-F}\right)\right)$ & $\ln (t)$ \\
Weibull & $\ln \left(\ln \left(\frac{1}{1-F}\right)\right)$ & $\ln (t-\gamma)$ \\
Three-parameter & $\emptyset^{-1}(F)$ & $t$ \\
Weibull & $\emptyset^{-1}(F)$ & $\ln (t)$ \\
Normal & $\ln \left(\ln \left(\frac{1}{F}\right)\right)$ & $t$ \\
Log-normal & $\ln \left(\ln \left(\frac{1}{1-F}\right)\right)$ & $t$ \\
value & & $t$ \\
Smallest extreme \\
value
\end{tabular}


In the MINITAB statistical software program, the Anderson-Darling (A-D) statistic was utilized to test the goodness of fitting, by measuring of how far plot points fall from the fitted line, in a probability plot. This process helps to determine the best fitted distribution function to the fatigue lifetime data at each stress level. Substantially, for comparing fitting of several distributions to data, the one with the lowest value of A-D statistic has best fitting to experimental data [24]. However, the A-D statistics, calculated for different distributions, may not be directly comparable or it is not enough for comparing when the A$D$ values are close, since A-D values are distributed differently for different distribution functions. To have a better comparison for the fit evaluation of different distribution functions, an additional criterion such as the P-value should be utilized [24]. The P-value is a probability that measures the evidence against the null hypothesis that data follow the distribution. For an A-D test, the null hypothesis is that data follow the distribution. Therefore, higher values of the P-value are associated with a better fitting distribution to the data [24]. The Pvalue should be compared for each distribution function to the significance level. Generally, a significance level (denoted as $\alpha$ ) of 0.05 works well. A significance level of 0.05 shows a $5 \%$ risk of concluding data do not follow the distribution, when they actually do follow the distribution [24]. If the P-value is less than or equal to the significance level $(P \leq \alpha)$, the decision is to reject the null hypothesis and conclude that the data do not follow the distribution. If the $\mathrm{P}$-value is greater than the significance level $(P>$ $\alpha)$, the decision is to fail to reject the null hypothesis [24]. Hence, to assess the appropriateness of a distribution fit, the A-D value and P-value must be considered. For comparing the fit of several distributions, the one with the largest P-value and the lowest A-D value generally has best fitting to experimental data [24].

\subsection{Fatigue limit testing}

The aim of fatigue limit testing (also called the fatigue strength or endurance test) is to evaluate a statistical distribution of the fatigue limit at a specific high-cycle fatigue lifetime. The staircase test methodology is the most recommended one among many fatigue limit test methods that has been adopted by many standards to investigate the statistical behavior of a fatigue limit. Hence, staircase fatigue tests for the fatigue limit of the EN-GJS700-2 ductile cast iron were conducted by cyclic loading, considering $R_{\sigma}=-1$.

Fatigue endurance tests are aborted by the specimen fail, which represents a complete fracture of specimens or when specimens have sustained at $2 \times 10^{6}$ cycles; then, they are considered as run-outs. It should be noted that the specific fatigue lifetime, mentioned above, was also considered in the literature [11] and by the data bank in the FEMFAT software [25].

According to the staircase method [4], the first test was conducted at a predetermined stress level. Then, if the specimen fails prior to the lifetime of interest, the stress level is decreased one step for the next test. Otherwise, if the specimen does not fail within the lifetime of interest, one step higher stress level should be utilized for the following test. Therefore, each test is dependent on previous test results. Tests continue until a valuable number of data are obtained to predict the fatigue endurance limit. Totally, 7 fatigue limit tests with standard specimens, which were extracted from engine crankshaft, were implemented. In addition, stress levels were considered at 60,55 and $50 \mathrm{~N}$. Therefore, the stress increment of $5 \mathrm{~N}(18.88 \mathrm{MPa})$ was utilized.

The Dixon-Mood method [4], based on the MLE was implemented as a typical data reduction technique to obtain statistical parameters of test results, according to a normal distribution. This method evaluates the mean value $\left(\mu_{s}\right)$ and the standard deviation $\left(\sigma_{s}\right)$ of a fatigue limit $\left(S_{e}\right)$, through approximate formulations. The equally spaced stress levels $\left(S_{i}\right)$ are numbered by the index, $i$, with a selected increment, $d$. The lowest stress level $\left(S_{0}\right)$ is denoted by $i=0$. Two values, $C$ and $D$ could be specified by determining the number of the less frequent event $\left(n_{i}\right)$ at the numbered stress level $i$ [4], as follows,

$$
\begin{aligned}
& C=\sum i \times n_{i} \\
& D=\sum i^{2} \times n_{i}
\end{aligned}
$$

The mean fatigue limit is then calculated by the following equation [4],

$$
\mu_{s}=S_{0}+d \times\left(\frac{C}{\sum n_{i}} \pm 0.5\right)
$$

The plus sign ( + ) is utilized, if the survival event is less repeated. If the failure event is less repeated, the minus sign (-) is used.

The standard deviation could be evaluated by following formulations [4],

$$
\begin{aligned}
& \sigma_{s}=1.62 \times d \times\left[\frac{D \times \sum n_{i}-C^{2}}{\left(\sum n_{i}\right)^{2}}+0.029\right] \\
& \text { if } \frac{D \times \sum n_{i}-C^{2}}{\left(\sum n_{i}\right)^{2}} \geq 0.3
\end{aligned}
$$

or

if $\frac{D \times \sum n_{i}-C^{2}}{\left(\sum n_{i}\right)^{2}}<0.3$.

$$
\sigma_{s}=0.53 \times d
$$

It should also be emphasized that the stress increment value should be in the range of $0.5 \sigma_{s}-2.0 \sigma_{s}$ [4].

The confidence interval value could be determined as follows [4],

$$
S_{e}=\mu_{s} \pm Z \times \sigma_{s}
$$

It should be mentioned that the $Z$ value, in the above equation, is the $p^{\text {th }}$ percentile of the standard normal distribution and associated with the confidence level. When the confidence level was considered as 99, 95 and $90 \%$, the $Z$ value was $2.576,1.960$ and 1.645 , respectively [4]. Besides, it should be again noted that the mean value of the fatigue limit $\left(\mu_{s}\right)$ and the standard deviation $\left(\sigma_{s}\right)$ were obtained for a normal distribution.

\section{Results and Discussions}

\subsection{Distribution functions analysis}

Selecting an appropriate distribution function is an essential first step in performing the reliability analysis 
[24]. If the selected distribution does not fit the data well, then the reliability estimation will be inaccurate. Hence, according to experimental data, the goodness of fitting through most commonly used distribution functions, such as Weibull (two- and three-parameter), normal, lognormal, smallest extreme value and extreme maximum value functions, were checked. Based on the A-D statistical method, six distribution functions were compared and obtained results are illustrated in Table 4, for corresponding stress levels, including 110 and $60 \mathrm{~N}$, for samples of obtained results. In addition, the rank ( 1 to 6) of each distribution function at each stress level, due to evaluate the goodness of fitting, is shown in Table 5. In this figure, the averaged rank of distribution functions was also determined to find the best function for estimating the reliability performance. As can be seen in Tables 4 and 5, the three-parameter Weibull distribution function was generally the best fitted probability function to the experimental fatigue lifetime at different stress levels. Besides, the extreme maximum value function had the worst fitting effect, in this case study. Therefore, the three-parameter Weibull distribution was selected for ENGJS700-2 fatigue strength data fitting, due to its adaptability to experimental data. Such function was also recommended in the literature [9], for the crankshaft. As mentioned before, all calculations were performed by the MINITAB software. Table 6 depicts parameters of the three-parameter Weibull distribution function with standard error and 95\% confidence level at different stress levels.

The cumulative distribution curve, contains the relation between the failure probability and fatigue lifetime of the studied material, is predicted by the three-parameter Weibull previously chosen from six kinds of distributions. This curve including the cumulative probability versus the fatigue lifetime at different stress levels is shown in Figure 1. As can be seen from Figure 1, the percentage of failures was greater at higher values of the fatigue lifetime. As a sample plot, the cumulative probability versus the fatigue lifetime at a constant stress level (at 60 $\mathrm{N})$ with scatter-bands can be seen in Figure 2, for the confidence level of 99,95 and $90 \%$.

Table 4. The A-D value and the P-value for fitting of distribution functions.

\begin{tabular}{ccccc}
\hline \multirow{2}{*}{ Distributions } & \multicolumn{2}{c}{$110 \mathrm{~N}$} & \multicolumn{2}{c}{$60 \mathrm{~N}$} \\
\cline { 2 - 5 } & A-D & P-value & A-D & P-value \\
\hline Two-parameter Weibull & 0.33 & $>0.25$ & 0.41 & $>0.25$ \\
Three-parameter Weibull & 0.28 & $>0.50$ & 0.28 & $>0.50$ \\
Normal & 0.24 & 0.54 & 0.44 & 0.20 \\
Log-normal & 0.23 & 0.58 & 0.28 & 0.52 \\
Extreme maximum value & 0.25 & $>0.25$ & 0.36 & $>0.25$ \\
Smallest extreme value & 0.34 & $>0.25$ & 0.60 & 0.10 \\
\hline
\end{tabular}

Table 5. The rank of distribution functions.

\begin{tabular}{cccccccc}
\hline \multirow{2}{*}{ Distributions } & \multicolumn{6}{c}{ Stress levels (N) } & Averaged \\
\cline { 2 - 7 } & 110 & 100 & 90 & 80 & 70 & 60 & rank \\
\hline Two-parameter Weibull & 5 & 3 & 6 & 5 & 3 & 4 & 4.33 \\
Three-parameter Weibull & 4 & 1 & 4 & 2 & 1 & 1 & 2.16 \\
Normal & 2 & 4 & 1 & 3 & 4 & 5 & 3.16 \\
Log-normal & 1 & 5 & 2 & 6 & 5 & 2 & 3.50 \\
Extreme maximum value & 3 & 6 & 5 & 4 & 6 & 3 & 4.50 \\
Smallest extreme value & 6 & 2 & 3 & 1 & 2 & 6 & 3.33 \\
\hline
\end{tabular}

Table 6. Parameters of the three-parameter Weibull distribution function for the experimental data for $95 \%$ confidence level, as a sample for obtained results.

\begin{tabular}{ccccc}
\hline Stress & \multirow{2}{*}{ Level } & Parameter & \multirow{2}{*}{ Estimate } & \multicolumn{2}{c}{$95 \%$ Confidence Level } \\
\cline { 4 - 5 } 110 & Shape & 3.81 & 1.79 & Upper \\
$\mathrm{N}$ & Scale & $1.66 \mathrm{E}+3$ & 1260.97 & 2176.59 \\
& Location & $5.13 \mathrm{E}+3$ & 5125.20 & 5125.20 \\
& Shape & 8285.00 & 749.96 & 91527.10 \\
100 & Scale & $6.02 \mathrm{E}+6$ & 632710.00 & 57328757.00 \\
$\mathrm{~N}$ & Location & $-6.01 \mathrm{E}+6$ & -19580380.00 & 7561027.00 \\
& Shape & 1557.21 & 672.70 & 3604.77 \\
90 & Scale & $1.07 \mathrm{E}+7$ & 10709754.00 & 10723905.00 \\
$\mathrm{~N}$ & Location & $-1.07 \mathrm{E}+7$ & -10677269.00 & -10677269.00 \\
& Shape & 886.62 & 377.57 & 2081.98 \\
80 & Scale & $2.83 \mathrm{E}+7$ & 28279349.00 & 28344572.00 \\
$\mathrm{~N}$ & Location & $-2.82 \mathrm{E}+7$ & -28161329.00 & -28161329.00 \\
& Shape & 2856.59 & 16.37 & 493444.00 \\
70 & Scale & $1.22 \mathrm{E}+8$ & 745140.00 & $1.97 \mathrm{E}+10$ \\
$\mathrm{~N}$ & Location & $-1.21 \mathrm{E}+8$ & -737672475.00 & 496053350.00 \\
& Shape & 1.54 & 0.87 & 2.71 \\
& Scale & $5.35 \mathrm{E}+5$ & 321029.00 & 892119.00 \\
& Location & $3.81 \mathrm{E}+5$ & 380746.00 & 380746.00 \\
\hline
\end{tabular}

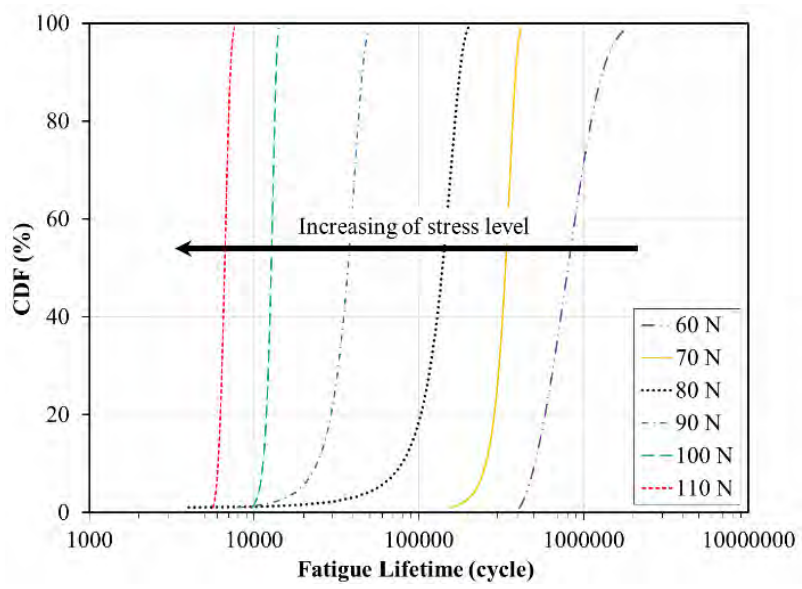

Fig. 1. The cumulative probability versus the fatigue lifetime at different stress levels.

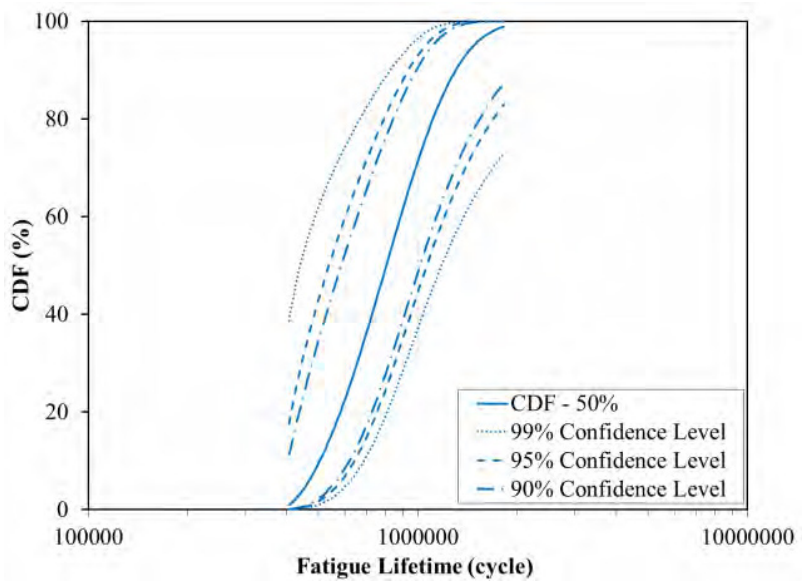

Fig. 2. The cumulative probability versus the fatigue lifetime with scatter-band at $60 \mathrm{~N}$ of the stress level for the confidence level of 99,95 and $90 \%$. 


\subsection{Fatigue reliability analysis}

The term "reliability" in engineering refers to the probability that a product, or a system, will perform its designed functions under a given set of operating conditions, for a specific period of the time. The reliability is also known as the "probability of survival" [19].

Using the MLE method and considering the threeparameter Weibull distribution function, Figure 3 illustrates a reliability plot for different stress levels for the EN-GJS700-2 ductile cast iron. In Figure 4, as a sample plot, the reliability versus the fatigue lifetime can be seen at a constant stress level (at $60 \mathrm{~N}$ ) with scatterbands, for the confidence level of 99,95 and $90 \%$.

These figures could be a considerable value for designers, where the fatigue lifetime could be easily determined at any degree of the reliability for such material. For example, if a value of $90 \% \mathrm{R} 95 \% \mathrm{C}$ is used for the component design, this particular value indicates that there is a $90 \%$ possibility of the survival (or the reliability) with a $95 \%$ confidence level for a fatigue lifetime at a specified stress level. Besides, Figure 3 shows that the trend of the reliability loss by increasing the fatigue lifetime, under constant amplitude loading.

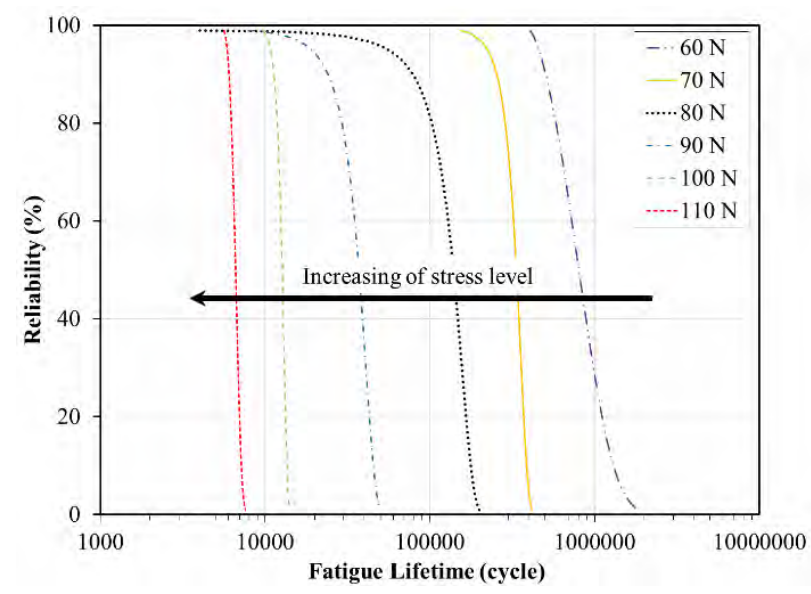

Fig. 3. The reliability versus the fatigue lifetime at different stress levels.

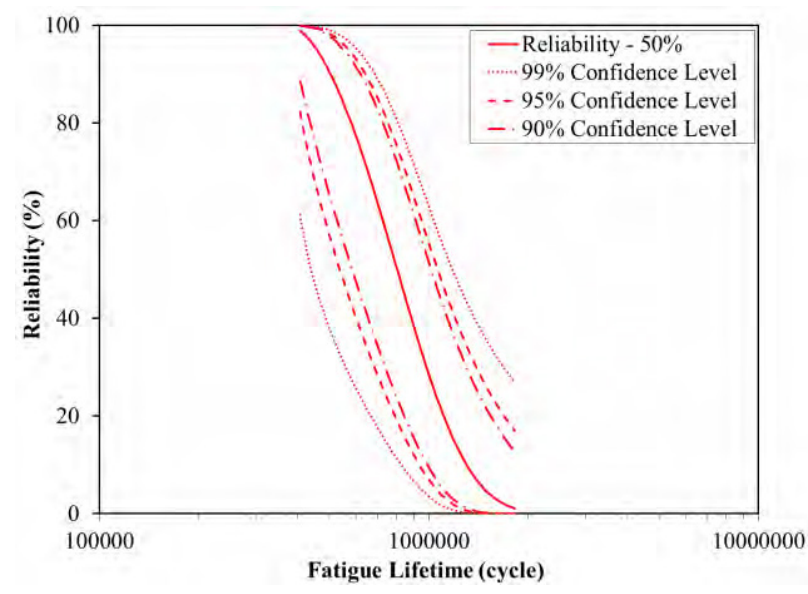

Fig. 4. The reliability versus the fatigue lifetime with scatterband at $60 \mathrm{~N}$ of the stress level, with the scatter-band for the confidence level of 99,95 and $90 \%$.
In addition, these results are fundamental for the estimation of the optimal warranty period and for sizing of the supply system involved with the service lifetime. Once that the reliability period is known, the manufacturer could plan and manage all activities, necessary for the service lifetime. In this way, costs would be reduced, while ensuring a high quality of the service offered [10].

A careful analysis of these reliability plots indicated that the reliability remained higher (almost constant) for the initial period and later on, started declining with the usage cycle. This phenomenon explained existing understanding of periods of the crack initiation and the crack propagation. It should be noted that he reliability varied by changing the stress level. It is also clear from these plots that the crack initiation period was smaller for higher stress levels and the reliability loss was faster, during the crack propagation, indicating a faster degradation or a higher rate of the damage accumulation. However, when a product is subjected to the high stress, the rate of the reliability loss is higher and starts at the early stage of the lifetime. This happens due to a shorter crack initiation period under high stress levels and relatively a longer crack propagation period [14].

\subsection{S-N curve and scatter-band analysis}

The Analysis of the S-N curve was performed for the purpose of determining constant values (or properties) of the material during the high-cycle fatigue regime. Figure 5 shows series of S-N diagrams for the EN-GJS700-2 ductile cast iron, with various values of the confidence level at $90 \%$ of the reliability. Obtained results in this figure indicate that the scatter-band in the fatigue lifetime became wider at $10^{5}$ to $10^{6}$ cycles. A similar behavior was also observed by the literature [19], for composite materials under rotating bending fatigue testing.

In general, the variance of the logarithmic lifetime increased, as the stress level decreased. It has been observed that once cracks nucleated in the material at high stress levels, these cracks had a better chance of overcoming the surrounding microstructure. Therefore, there was a long period to propagate. Most cracks could successfully nucleate at low stress levels, but only few cracks could overcome surrounding obstacles to grow. Thus, the crack propagation period was so small [4]. For the S-N curve, a relation between the fatigue lifetime $\left(N_{f}\right)$ and the stress amplitude $\left(\sigma_{a}\right)$ could be written [1], as follows,

$$
\sigma_{a}=\sigma_{f}^{\prime}\left(2 N_{f}\right)^{b}
$$

Where $\sigma_{f}^{\prime}$ and $b$ are the fatigue strength coefficient and the fatigue strength exponent, respectively. Obtained results based on these material constants for the ENGJS700-2 ductile cast iron can be observed in Table 7, based on $90 \%$ of the reliability and various confidence levels. It should be noted that in Figure 5 and Table 7, there was no data for the lower-band of $99 \%$ confidence level; therefore, there was no line for the scatter-band. 


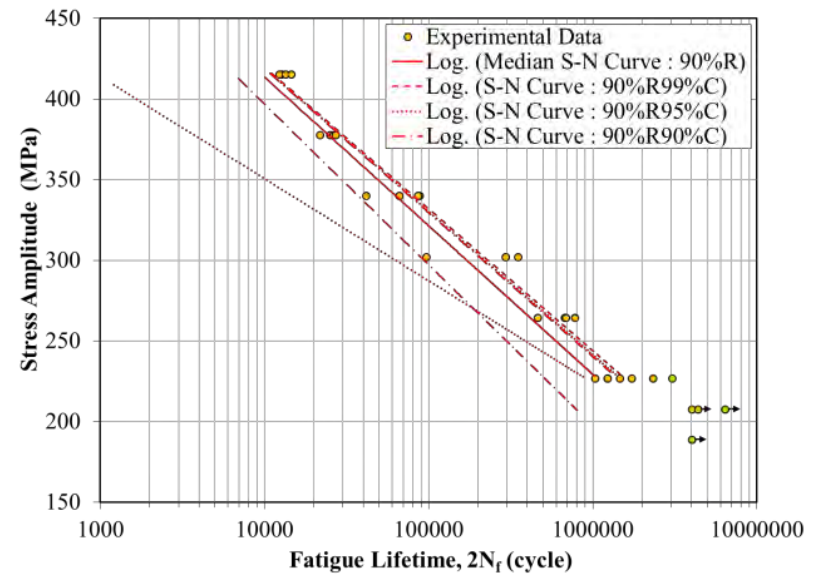

Fig. 5. The S-N curve and scatter-bands for different confidence levels in the EN-GJS700-2 ductile cast iron.

Table 7. The characterization of the high-cycle fatigue behavior in the EN-GJS700-2 ductile cast iron.

\begin{tabular}{|c|c|c|c|}
\hline $\begin{array}{l}\text { Reliability }(\mathrm{R}) \text { and } \\
\text { confidence level } \\
(\mathrm{C})\end{array}$ & $\mathrm{R}^{2}$ & $\begin{array}{l}\text { Fatigue strength } \\
\text { coefficient } \\
(\mathrm{MPa})\end{array}$ & $\begin{array}{c}\text { Fatigue } \\
\text { strength } \\
\text { exponent (-) }\end{array}$ \\
\hline Median S-N Curve & 0.9885 & 1378.1611 & -0.1283 \\
\hline $\begin{array}{c}90 \% \mathrm{R} 99 \% \mathrm{C} \\
\text { (Upper) }\end{array}$ & 0.9919 & 1322.5131 & -0.1218 \\
\hline $\begin{array}{c}90 \% \text { R99\%C } \\
\text { (Lower) }\end{array}$ & - & - & - \\
\hline $\begin{array}{c}90 \% \mathrm{R} 95 \% \mathrm{C} \\
\text { (Upper) }\end{array}$ & 0.9916 & 1334.7502 & -0.1230 \\
\hline $\begin{array}{c}90 \% \text { R95\%C } \\
\text { (Lower) }\end{array}$ & 0.4547 & 809.4685 & -0.0929 \\
\hline $\begin{array}{c}90 \% \mathrm{R} 90 \% \mathrm{C} \\
\text { (Upper) }\end{array}$ & 0.9917 & 1340.9111 & -0.1236 \\
\hline $\begin{array}{c}90 \% \mathrm{R} 90 \% \mathrm{C} \\
\text { (Lower) }\end{array}$ & 0.8924 & 1457.1357 & -0.1408 \\
\hline
\end{tabular}

As it can be observed from obtained results in Table 7, high-cycle fatigue properties (including the fatigue strength coefficient and the fatigue strength exponent) of the material varied, when the confidence level changed, both at the lower and upper scatter-band. It should be mentioned that the coefficient of determination $\left(\mathrm{R}^{2}\right)$ was more than $89 \%$, unless one data point for the confidence level of $95 \%$, which generally showed proper modeling to obtain fatigue properties.

\subsection{Fatigue limit assessment}

In accordance with mentioned steps in Part 2.3, the fatigue endurance limit of the EN-GJS700-2 ductile cast iron was experimentally examined. Figure 6 illustrates results of the fatigue endurance limit, including failure/run-out records of the studied material, at different confidence levels, including values of 90,95 and $99 \%$. It should be noted that in Figure 6, black dots and open circles represented test data, failed prior to $2 \times 10^{6}$ cycles and suspended at $2 \times 10^{6}$ cycles, respectively. Besides, red lines represents the mean and the interval (or the scatterband) of the endurance limit at different confidence levels. In addition, all determined values are also listed in Table 8, including the mean endurance limit, the standard deviation and lower and upper scatter-band of the endurance limit at different confidence levels.

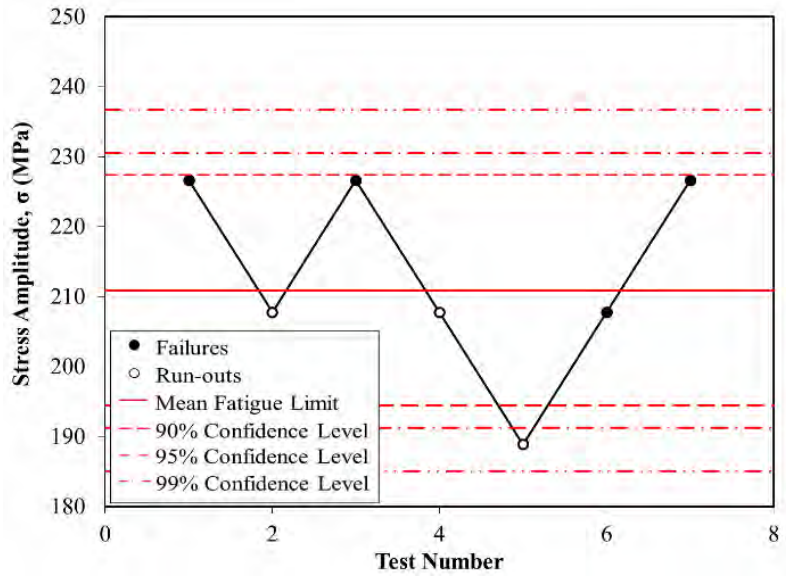

Fig. 6. Results of the Dixon-Mood analysis for the ENGJS700-2 ductile cast iron.

Table 8. Results of fatigue limit testing for the EN-GJS700-2 ductile cast iron.

\begin{tabular}{ccc}
\hline Fatigue limit properties $(\mathrm{MPa})$ & $\begin{array}{c}\text { Confidence } \\
\text { level }\end{array}$ & $\begin{array}{c}\text { Experimental } \\
\text { data }\end{array}$ \\
\hline Mean fatigue limit $\left(\mu_{s}\right)$ & - & 210.899 \\
Fatigue limit scatter-band & $90 \%$ & $194.433-227.365$ \\
Fatigue limit scatter-band & $95 \%$ & $191.280-230.519$ \\
Fatigue limit scatter-band & $99 \%$ & $185.114-236.685$ \\
Standard deviation $\left(\sigma_{s}\right)$ & - & 10.010 \\
Stress increment $(d)$ & - & 18.887 \\
$d / \sigma_{s}$ & - & 1.880 \\
\hline
\end{tabular}

As it can be seen in Table 8 , the stress increment value was equal to $1.88 \sigma_{s}$, which was in the recommended range in the literature [4]. In addition, the mean fatigue endurance limit measured for the studied material was about $211 \mathrm{MPa}$. The fatigue limit of the studied material in the FEMFAT data bank was $240 \mathrm{MPa}$ [25]. As the first discussion in this section, the mean fatigue limit value was $12 \%$ lower than the endurance limit from the FEMFAT data bank. For the reason, it could be stated that the endurance limit in the FEMFAT data bank was determined by standard specimens, made from the material, and not from the component. However, presented experimental data were conducted by standard specimens, extracted from the crankshaft web, due to consider the manufacturing effect. This emphasizes that the manufacturing effect could decrease the fatigue strength of the raw material. Additionally, as seen in Figure 6 and Table 8, when a higher value for the confidence level was utilized, a wider scatter-band was obtained. It is obvious that to have higher reliable confidence intervals, higher degrees of the confidence level should be considered.

\section{Conclusions}

In the present research, the reliability prediction, the S-N curve, the scatter-band analysis and the fatigue limit assessment of high-cycle fatigue properties in the ENGJS700-2 ductile cast iron were performed. For this objective, experimental data, acquired from four-point rotary bending fatigue testing on standard specimens (extracted from engine crankshafts) were analyzed. 
Obtained results could be listed as follows,

- Based on the distribution functions analysis, the three-parameter Weibull distribution was optimum for the experimental data adaptation of the studied material.

- The reliability of the material was calculated at each stress level, considering the threeparameter Weibull distribution.

- The scatter-band for the S-N curve at different confidence levels was also obtained to find highcycle fatigue properties of the material.

- The fatigue limit of the studied material was obtained as $211 \mathrm{MPa}$, considering the effect of manufacturing approaches in the component, based on the staircase test method.

As the acknowledgement, Authors would like to thank Irankhodro Powertrain Company (IPCo) for financially supporting of the presented work.

\section{References}

1. M.J. Khameneh, M. Azadi, Eng. Fail. Anal., 85 (2018) 189-200

2. Metallic materials - Fatigue testing Statistical planning and analysis of data, Standard No. ISO 12107, ISO International Standard, (2012)

3. J.M. Zhai, X.Y. Li, Int. J. Fatigue, 44 (2012) 107115

4. Y.L. Lee, J. Pan, R. Hathaway, M. Barkey, Fatigue Testing and Analysis, John Wiley and Sons, (2004)

5. P.D. Toasa Caiza, T. Ummenhofer, Int. J. Fatigue, 106 (2018) 70-80

6. A.K. Chandrappa, K.P. Biligiri, Const. Build. Mater., 153 (2017) 1-15

7. S.P. Zhu, Q. Liu, H.Z. Hong, Struct. Integ. Proc., 4 (2017) 3-10

8. L. Feng, M.L. Yu, N.G. Fang, L.L. Juan, Const. Build. Mater., 95 (2015) 207-217

9. X. Chen, X. Yu, R. Hu, J. Li, Eng. Fail. Anal., 42 (2014) 210-220

10. O. Borgia, F.D. Carlo, N. Fanciullacci, M. Tucci, Accelerated life tests for new product qualification: a case study in the household appliance, $11^{\text {th }}$ IFAC Workshop on Intelligent Manufacturing Systems, Brazil, (2013)

11. G. Cevik, R. Gurbuz, Eng. Fail. Anal., 27 (2013) 250-261

12. J. Klemenc, M. Fajdiga, Int. J. Fatigue, 43 (2012) 90-97

13. S. Mohd, Y. Mutoh, Y. Otsuka, Y. Miyashita, T. Koike, T. Suzuki, Eng. Fail. Anal., 22 (2012) 64-72

14. V. Rathod, O.P. Yadav, A. Rathore, R. Jain, Int. J. Qual., Statis. Reliability, (2011) doi:10.1155/2011/718901 (to be published)

15. C. Bouraoui, R. Ben Sghaier, R. Fathallah, Mater. Des., 30 (2009) 475-486

16. R.B. Sghaier, C. Bouraoui, R. Fathallah, T. Hassine, A. Dogui, Int. J. Fatigue, 29 (2007) 209-221

17. P. Spiteri, S. Ho, Y.L. Lee, Int. J. Fatigue, 29 (2007) 318-329
18. J. Schijve, Int. J. Fatigue, 27 (2005) 1031-1039

19. U.A. Khashaba, J. Compos. Mater., 37 (4) (2003) 317-331

20. M.H.H. Shen, Int. J. Fatigue, 21 (1999) 699-708

21. R.H. Doremus, J. Appl. Phys., 54 (1983) 193-198

22. Standard practice for statistical analysis of linear or linearized stress-life $(S-N)$ and strain-life $(\varepsilon-N)$ Fatigue Data, Standard No. ASTM E739-10, ASTM International, (2015)

23. G. Yang, Life cycle reliability engineering, John Wiley and Sons, USA, (2007)

24. MINITAB software, Help of the software

25. FEMFAT software, Help of the software 